\title{
Optical chemical sensor for solving gas analysis tasks
}

\author{
Aleksandr Maslennikov ${ }^{1, *}$, Ilya Zubkov $^{2}$, S. Kovalenko ${ }^{3}$ \\ ${ }^{1}$ Nizhny Novgorod State Technical University n.a. R. E. Alekseev, 603950, Minina str., 24, Nizhny \\ Novgorod, Russia \\ ${ }^{2}$ Dzerzhinsk Polytechnic Institute, Nizhny Novgorod State Technical University n.a. R. E. Alekseev, \\ 606039, Gaidara str., 49, Dzerzhinsk, Russia \\ ${ }^{3}$ Bulat LLC, 121471, Ryabinovaya str., 26, Moscow, Russia
}

\begin{abstract}
A brief review of the principles of constructing optical gasanalyzing sensors is presented. It is noted that the influence of humidity of the surrounding gas environment during gas analytical procedures using solid-state gas analytical sensors is a serious technological problem. It is shown that sensors of particular interest are functioning on the principle of absorption of the primary light flux because of their reduced sensitivity to fluctuations in the humidity of the carrier gas. The design and the gas analytical properties of an optical chemical sensor are described. Ammonia was used as a test substance in the studies and as a sensitive sensor coating; a functional polymer having a specific highly selective reaction to ammonia was used. The static properties of the sensor were determined, the effect of the carrier gas humidity on its output signal, as well as the stability of the gas analytic properties of the sensor when interacting with ammonia. Analysis of the properties of the optical chemical sensor allows us to conclude that the proposed sensor is promising for solving various gas analytical problems.
\end{abstract}

\section{Introduction}

One of the main types of solid-state gas-analytical chemical sensors are optical. Usually, the following principles of their action are singled out [1,2]:

- $\quad$ absorption of the primary light flux;

- $\quad$ reflecting primary light flux;

- luminescence (generation of secondary light flux).

In the work of sensors, the dependencies of the optical properties of solid media on the concentrations of the gaseous substances are used.

\footnotetext{
* Corresponding author: mavdoc@ rambler.ru
} 
The absorption of the primary optical radiation depends on the structure and the aggregate state of the solid, the thickness of the layer, the wavelength of the light flux, and other factors, and is described by the Bouguer-Lambert and Behr's laws.

According to the Bouguer-Lambert law, for a homogeneous medium with an absorption coefficient $\alpha_{\mathrm{absorb}}$, and the thickness of its layer $L$, being perpendicular to the primary monochromatic light flux with intensity I0, the интенсивность прошедшего светового потока $I$ equals:

$$
\mathrm{I}=\mathrm{I}_{0} \exp \left(-\alpha_{\text {ĭ̃äe }} L\right) .
$$

The coefficient $\alpha_{\text {absorb }}$ can take into account scattering, in determining the attenuation of the intensity of light passing through the medium.

According to Behr's law, the absorption is proportional to the number of particles of the absorbing substance $N$ :

$$
\alpha=\sigma N
$$

where $N$ is the concentration of a transparent substance; $\sigma$ is the cross-section for the absorption of the translucent substance at a given wavelength of the light flux.

When both laws are executed, the combined Bouguer-Lambert-Behr's law will be valid:

$$
\mathrm{I}=\mathrm{I}_{0} \exp (-L \sigma N)
$$

In the case of a change in the concentration $N$ of the transilluminated substance along the path of propagation of the light flux, the integral form of the Bouguer-Lambert-Behr's law:

$$
\mathrm{I}=\mathrm{I}_{0} \exp \left[-\int_{0}^{L} \sigma N(z) d z\right],
$$

where $N(\mathrm{z})$ is distribution of the concentration of the transilluminated substance along the $\mathrm{z}$ axis.

In the construction of optical sensors based on the reflection of the primary light flux, the dependence of the reflected light intensity on the electronic structure of atoms, molecules, and ions in the surface layer of the substance, the absorption processes and multiple scattering in it, and its dependence on the wavelength of the incident light are used. This allows the reflection effect to be used to study the composition and structure of surface layers of solids and turbid media, and also to identify adsorbed compounds.

The use of luminescence, which is a luminescence of a substance arising after the absorption of external excitation energy, is excessive in comparison with thermal radiation. Photoluminescence is characterized by energy and quantum yield, kinetics, polarization, and absorption and luminescence spectra. Usually photoluminescence is excited by ultraviolet radiation. The photoluminescence parameters allow one to judge the presence of certain substances and their concentration in the samples under study.

For the construction of gas analytical sensors, the absorption (absorption) of the primary luminous flux is most often used. Moreover, such absorption is possible in the outer space, near the optical wave propagation layer, as occurs in integrated optical chemical sensors [3, 4]. In addition, the absorption can occur in a special film, changing its properties (color, transparency) when interacting with the analyzed gaseous component [5]. As a sensitive compound in the optical chemical sensor used functional polymer, which is immobilized on the polystyrenesulfonate sodium acridine dye. A specific feature of this compound is the ability to quickly and reversibly change the color and transparency of its film, being about 1 micron thick, from blue to light yellow and back, respectively, when ammonia gas is supplied 
and removed from its surrounding atmosphere. This makes it possible to make it the basis of an optical chemical sensor.

\section{Problem Statement}

Thus, the existing connection makes it possible to use it as a sensitive coating of an optical chemical sensor. For initial studies, it was proposed to use a simple single-layer optical chemical sensor, which is translucent by light radiation with a wavelength of about $630 \mathrm{~nm}$, which corresponds to a red color. As a source of such radiation it is supposed to use a semiconductor LED. Preliminary studies of films of this compound showed their long-term resistance to daylight. Moreover, even exposure to direct sunlight for 100 hours did not cause noticeable discoloration of the polymer film. This fact suggests that the effect of the red LED radiation, even of medium power, will also not cause significant changes in the properties of the film under investigation, at least in comparable time intervals, which was confirmed by further studies. This, in turn, opens the way to creating a multilayer optical chemical sensor, having a higher sensitivity if compared to single-layered. In addition, the multilayer makes it possible to reduce the thickness of a single film of the multilayer structure, which gives reason to hope for an improvement in the speed of such a sensor.

As a receiver passed through the radiation film, a photodiode compatible with the maximum spectral sensitivity with an LED was used, the signal of which, after amplification, was applied to a 16-bit analog-to-digital converter and then to an interface converter (USB). Then the signal was fed to a personal computer, where it was stored and processed.

To construct a gas analytical optical chemical sensor for ammonia based on the functional polymer in question, it is necessary to determine its basic gas analytical properties, which primarily determine sensitivity and stability when it is used multiple times.

For this, it is necessary to determine the static characteristics of the developed sensor under various operating conditions. To assess the sensitivity of the sensor to temperature, which will allow you to determine both the operating temperature of the sensor and the need for temperature control of the sensor during operation. In addition, it is important to assess the stability of the sensor and the reproducibility of its signal.

Thus, the main purpose of this study is to evaluate the capabilities of an optical ammonia sensor having one layer of a sensitive coating to further build on its basis an ammonia gas analyzer with a threshold sensitivity of $2-5 \mathrm{mg} / \mathrm{m} 3$, which will allow for a reliable control of its concentration in the atmosphere at level of MPCs.

\section{Research Methods}

To explore the sensor, an experimental setup was assembled, the circuit of which is shown in Fig. 1. The setup allows one to determine the sensor response to various concentrations of ammonia at different temperatures and humidity of the gas mixture being analyzed. The temperature value is set and stabilized by the unit 9 . In addition, the temperature, as well as the humidity content of the gas mixture, was controlled by the Iva-6B2-K thermo-moisture. 


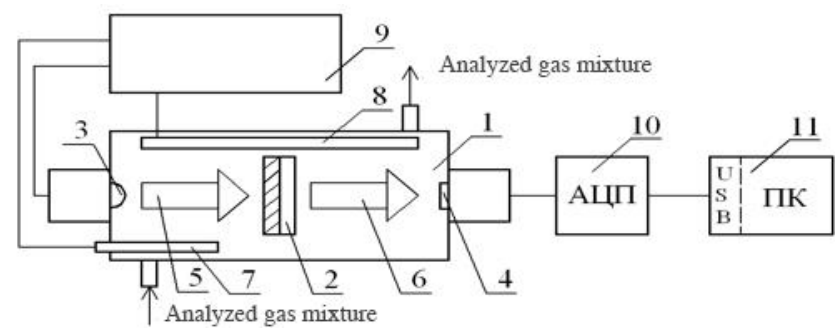

Fig. 1. The Experimental installation for the study of an optical chemical ammonia sensor: 1 - camera, 2 - sensor to be investigated, 3 - LED, 4 - photodiode, 5 - radiation flux to sensor, 6 - radiation flux after sensor, 7 - Thermal-moisture-sensor, 8 - heating element, 9 - monitoring and control unit, $10-$ analog-to-digital converter, 11 - personal computer.

The necessary concentration of ammonia in the chamber was created by supplying the necessary volumetric doses of an ammonia-air mixture of known concentration to the research chamber. Similarly, the necessary moisture content of the mixture being analyzed was created. The volume of these doses is small, compared with the volume of the chamber. At first, the required humidity level was created in the chamber. After that, the required volume of ammonia-air mixture was introduced. Then the moisture level was finally determined.

The temperature in the chamber was set in the control and control unit. As a temperature regulator in the installation, a TRM 101 type PID controller is used, with a pulse output signal. For operation, the regulator is equipped with its own temperature sensor in the chamber. In addition, it is monitored by the sensor signal of the thermal-moisture-sensor. The regulator and the thermal-moisture-sensor are part of the block 9. The main elements of the installation that determine its performance are:

- an analog-to-digital converter ADS1110A0IDBVT;

- a source of light is the LED L-1543SRC-C;

- a photodetector is the photodiode VT90N1.

The optical chemical sensor (2) is in the chamber and is a glass plate of $20 \times 20 \mathrm{~mm}$. The plate was coated with $50 \mu \mathrm{l}$ of a $1 \%$ solution of the functional polymer in dimethylformamid, after evaporation of which, a polymer film with a thickness of about $1 \mu \mathrm{m}$ remained on the glass. The output signals from the photodiode are the input to the analog-to-digital converter and then to the personal computer, where they are processed in accordance with the specified algorithm of work being embedded in the application software. The application software provides visualization and processing of the received information, in a user-friendly form.

After obtaining the primary experimental information, its processing was carried out, which allowed obtaining a number of dependencies characterizing the ability of an optical chemical sensor to determine the presence of ammonia in the composition of the carrier gas entering the sensor chamber. The signal to the personal computer is received in the form of a 16-bit digital code. To make the output signal more visible, the authors use the voltage signal at the input of the analog-to-digital converter as an output signal, which is determined by its conversion characteristic.

\section{Findings}

The results obtained in the results are presented below in the corresponding figures.

\subsection{Sensor's statistical characteristics}


Figure 2 shows the direct experimental results obtained at the research facility in the form of a family of static sensor characteristics. The characteristics were obtained at operating temperatures in the range of $10^{\circ} \mathrm{C}$ to $50^{\circ} \mathrm{C}$, with a relative humidity of the gas mixture in the chamber of $50 \%$. The concentration of ammonia in the gas medium in the chamber was created in the range $0-200 \mathrm{mg} / \mathrm{m}^{3}$, which corresponds to gas contamination with ammonia at the level of 10 MPCs.

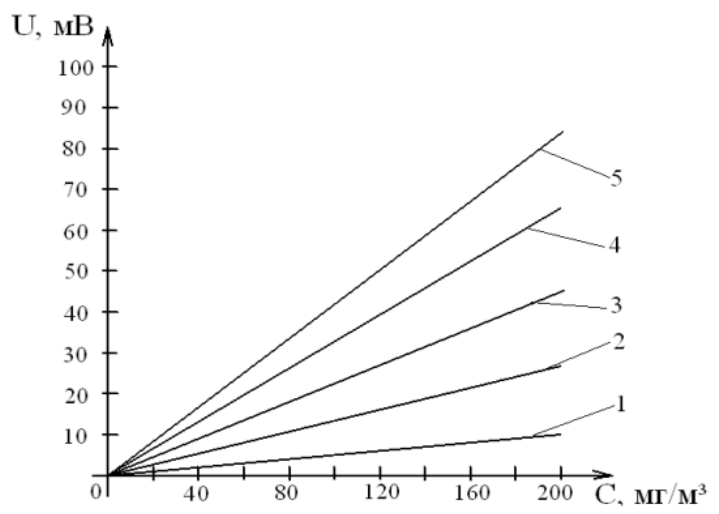

Fig. 2. Static characteristics of sensors at different temperatures: $1-10{ }^{\circ} \mathrm{C} ; 2-20^{\circ} \mathrm{C} ; 3-30{ }^{\circ} \mathrm{C} ; 4-40$ ${ }^{\circ} \mathrm{C} ; 5-50{ }^{\circ} \mathrm{C}$.

The characteristics obtained are of a character close to linear, which is explained by the low operating concentrations of ammonia and the lack of saturation of the sensor.

Figure 3 shows the sensitivity of the sensor to temperature.

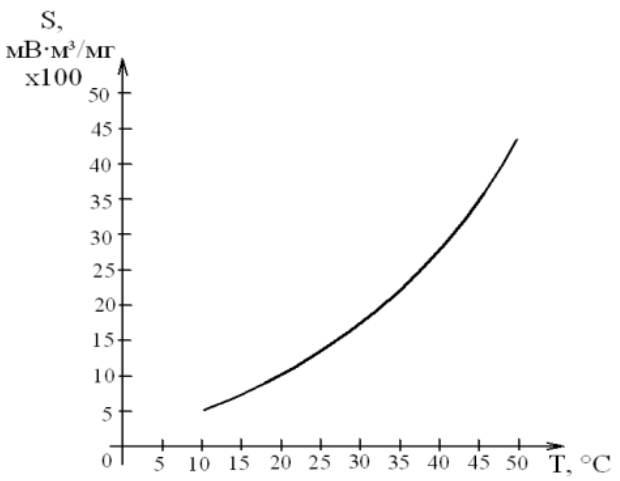

Fig. 3. Dependence of sensor sensitivity on temperature.

As can be seen, with increasing temperature, the sensitivity of the sensor increases significantly, which creates a wide field for maneuver while optimizing the design of the sensor and its operating conditions. Such optimization will be required when using a sensor in various gas analysis systems, including leak detection. The features of the use and capabilities of the optical chemical sensor under consideration in solving the leak tightness control problems have also been investigated and will be considered in the next article.

Here it can be noted, in particular, that as the temperature rises, the dynamic properties of the optical chemical sensor, which in most leak detection systems, are important, and in 
some applications, extremely important (for example, in the localization of leaks) should significantly change, in the direction of improvement.

\subsection{Effect of humidity on the sensor}

An important condition for the successful operation of any gas analyzer sensor that determines the concentration of something in ambient air is the low sensitivity of such a sensor to the humidity of the surrounding atmosphere, which is extremely unstable and can vary very widely in a very short time (for example, in the case of a shower in a hot summer day).

The dependence of the output signal of the sensor on the humidity of the gas in the chamber at different temperatures is shown in Fig. 4.

\subsection{Stability of sensor characteristics}

The stability of the sensor characteristics is determined by the stability of the sensitive coating. To evaluate it, a series of cycles was performed, purging the carrier gas with an ammonia concentration of $100 \mathrm{mg} / \mathrm{m}^{3}$ at different temperatures. The investigations were carried out for temperatures of $20^{\circ} \mathrm{C}, 35^{\circ} \mathrm{C}$, and $50^{\circ} \mathrm{C}$. For each temperature, 50 puffing/purging cycles were performed. Sensitivity of the sensor was determined, and this sensitivity was changed from cycle to cycle. The results obtained show that there is no significant change in the sensitivity of the sensor at such temperatures. Considering the stability of a sensitive coating at higher temperatures is not advisable because the temperature of the thermostating of sensors above $50^{\circ} \mathrm{C}$ is unlikely to be relevant.

In addition, one of the sensor samples was placed for 14 days in a sealed chamber, in which an atmosphere with an ammonia concentration of $500 \mathrm{mg} / \mathrm{m}^{3}$ was created. The chamber was stored at a temperature of about $22^{\circ} \mathrm{C}$. After 14 days, the sensor was removed, and its properties were again determined and then compared to the earlier defined values. The comparison showed the stability of static characteristics, which indicates that a prolonged contact of the functional polymer with ammonia does not lead to its destruction and loss of sensory properties.

\section{Conclusion}

The above dependences make it possible to conclude that the investigated sensor has a sufficiently high sensitivity to the concentration of ammonia in the carrier gas, with a relatively weak influence of the humidity of the analyzed gas on the final signal of the sensor.

Dependence of sensitivity to ammonia and humidity on temperature makes it important to introduce at least thermal compensation of the sensor signal and a better temperature control of the sensor at temperatures around $50^{\circ} \mathrm{C}$. Thermostating, in addition to stabilizing the sensor signal and increasing its sensitivity, allows the sensor to be protected from exposure to high humidity of the analyzed air, which will "automatically' decrease when the gas is heated to the thermostating temperature. However, the obvious disadvantage of introducing thermostating is the complication of both the hardware and software of the gas analyzer and the increase in its dimensions, weight, and power consumption.

The presented studies were carried out, in essence, on the simplest optical chemical sensor having only one gas sensitive layer of a functional polymer. To achieve higher gas analytical parameters and the sensitivity of the sensor to the values of MPCs, it is necessary to improve the construction of an optical chemical sensor. One of the ways can be the use of planar waveguide optical chemical sensors [6]. Obviously, other directions are possible for improving and optimizing the construction of an optical chemical sensor, an important one 
of which is the optimization of the design in terms of ensuring a higher speed of the optical chemical sensor.

The work was supported by the Ministry of Education and Science of the Russian Federation. Agreement № 14.577.21.0144 of November 28, 2014. The unique project ID is RFMEFI57714X0144.

\section{References}

1. G. Whitenett, G. Stewart, K. Atherton, B. Culshaw, W. Johnstone, J. Opt. A: Pure Appl. Opt., 5 (2003)

2. R. Wiesmann, L. Muller, R. Klein, A. Neyer, ECIO'95, Proceedings of 7th European Conference on Integrated Optics (Delft, 1995)

3. A. A. Egorov, M. A. Egorov, R. B. Smoliakov, T. K. Chekhlova, A. G. Timakin, Journal of Radio Electronics, 5 (2007)

4. A. A. Egorov, M. A. Egorov, Yu. I. Tsareva, and T. K. Chekhlova, Laser Physics, 17 (2007)

5. E. I. Soborovich, I. L. Zubkov, High-efficiency flat-waveguide structure of an optical chemical sensor for gas analysis (MGIEM, Moscow, 2002) 